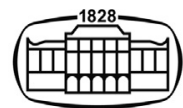

AKADÉMIAI KIADÓ

Journal of Behavioral Addictions

10 (2021) 3, 759-766

DOI:

10.1556/2006.2021.00049

(c) 2021 The Author(s)

\section{FULL-LENGTH REPORT}

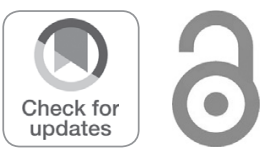

\title{
Linking temporal-parietal junction to internet addiction tendency: Moderating effect of critical thinking
}

\author{
DI LIU ${ }^{1 \dagger}$, YUHE MA ${ }^{1 \dagger}$, KAIXIANG ZHUANG ${ }^{2,3 \dagger}$, \\ QUNLIN CHEN $^{2,3}$, BAOGUO SHI $^{1 *} \odot$ and JIANG QIU ${ }^{2,3}$ \\ ${ }^{1}$ Beijing Key Laboratory of Learning and Cognition, School of Psychology, Capital Normal \\ University, Beijing 100048, China \\ ${ }^{2}$ Key Laboratory of Cognition and Personality (SWU), Ministry of Education, Chongqing 400715, \\ China \\ ${ }^{3}$ School of Psychology, Southwest University, Chongqing 400715, China
}

Received: December 13, 2020 • Revised manuscript received: March 28, 2021; May 12, 2021 • Accepted: July 7, 2021 Published online: August 31, 2021

\begin{abstract}
Backgrounds and aims: Internet addiction (IA) is a common internet-related addictive behavior. An enormous amount of previous research on IA disorders (IADs) have paid attention to the neural basis of abnormalities, while few studies have elucidated the neural distinctions of IA tendency in general population. Methods: The current study examined the neural basis of IA tendency combining with voxel-based morphometry (VBM) from the average student body $(N=244)$. Results: As the results presented, the gray matter density (GMD) of the left temporal-parietal junction (TPJ) was positively correlated with Internet Addiction Test (IAT) score. Further analysis revealed that critical thinking moderated the path between GMD in the TPJ and IA tendency. Specifically, the correlation between GMD in the TPJ and IA tendency was weaker for those with a higher critical thinking disposition. Discussion and conclusion: Higher critical thinking show a hindering effect in susceptibility to IA based on the neural basis of temporal-parietal junction differences.
\end{abstract}

\section{KEYWORDS}

internet addiction tendency, critical thinking, GMD, TPJ, moderating effect

\section{INTRODUCTION}

When a person spends most of their spare time engaging in video games, online shopping or online chat, internet addiction (IA) is considered because this behavior may lead to social difficulties and emotional problems. IA is one cannot control using the internet and may seem to be an impulse-control disorder and behavioral addiction (Holden, 2001; Young, 1998); it is linked with higher social anxiety, higher neuroticism and more depression (Wang, Ho, Chan, \& Tse, 2015; Yücens \& Üzer, 2018). In addition, with the rapid development of the network economy and constantly updated network technology, it is now known that $8.8 \%$ of China's teenagers experience IA (Xu et al., 2012). The present study aims to turn the perspective to the internet addiction tendency of healthy people, because it has become a very important social phenomenon and has attracted much attention from parents, teachers, schools and educational administration departments at all levels (Kuss \& Lopez-Fernandez, 2016).

Internet addiction will bring a lot of negative effects to the development of individual, family, and society. In previous studies, quite a few studies regarded it as internet addiction disorders or pathological internet use (Dong, Lu, Zhou, \& Zhao, 2011; Müller, Beutel, \& Wölfling, 2013). However, according to Diagnostic and Statistical Manual of Mental Disorder 
VI (DSM-VI), internet addiction has not been fully considered as a disease. Therefore, we suggest that internet addiction should be extended to an excessive internet use among the common people. While a large number of previous studies focused on internet addiction disorders (IADs), little paid attention on the excessive internet use or internet addiction tendency in a healthy sample. Several studies have shown that IAD is associated with cognitive impairment, which may lead to various behavioral and psychological abnormalities, attention dysfunction and neurological disorders and produce symptoms similar to drug addiction and pathological gambling (Weinstein \& Lejoyeux, 2010). Once these addicts stop engaging with the internet, they experience symptoms similar to drug withdrawal symptoms, such as restlessness, insomnia, anxiety, depression, mental dullness, and distraction. Attention-deficit/hyperactivity disorder (ADHD) has been indicated to be the most common comorbid psychiatric disorder of IAD. Yen, Ko, Yen, Wu, and Yang (2007) showed that there were obvious ADHD symptoms in those with IAD, and among them, attention dysfunction was the most relevant symptom (Yen et al., 2007). However, the brain regions associated with IAD when it is accompanied by attentional impairment and the possible underlying neural mechanisms remain to be studied. Attention is a psychological attribute that can be generated and used in learning, memory, judgment, executive function and many other cognitive processes by which resources are rationally allocated, while attentional dysfunction leads to impairments in a range of other higherorder functions. Corbetta and Shulman (2002) argued that there are two attentional orientation systems in the brain: one is the dorsal attention network (DAN), which is centered in the dorsal posterior parietal cortex and the dorsal frontal cortex, which is also known as the endogenous attentional system and participates in top-down attentiondirected control; the other is the ventral attention network (VAN), which is centered in the temporal-parietal junction (TPJ) and ventral frontal cortex, which is also known as the exogenous attention system and is involved in the detection of sensory-specific and behaviorally relevant stimuli related to bottom-up attention-directed control (Corbetta \& Shulman, 2002). Given the associations between IA and attention disorders, we suggest that individual differences in IA tendency may be related to brain regions associated with the attentional system.

In addition, a small number of studies have tried to investigate the neural basis of IA tendency. An earlier study using a comparative small sample size revealed that a negative correlation between frontal pole in gray matter volume (GMV) and IA tendency (Kühn \& Gallinat, 2014). Another study found that the GMV of dorsal frontal cortex in DAN and its connection with medial prefrontal cortex positively linked to internet addiction tendency ( $\mathrm{Li}$ et al., 2015). These results implied that the inhibitory control in top-down attentional system was disrupted or weaken. Recently, researchers suggested GMD is a major phenotype in brain structure (Gennatas et al., 2017), and it has a low correlation with GMV in youth and normal individuals.
GMV may not reveal the full picture of brain, due to the uncommon gray matter loss in youth and normal individuals (Gennatas et al., 2017; Gur et al., 2021). Based on this knowledge, the present study aimed to investigate the relation between IA tendency and GMD differences.

Researchers have come to believe that addiction are caused by an disequilibrium between two separate but interconnected systems in making decisions: impulsive systems and reflective systems. Actually, the cognitive processes involved in addictive behaviors or tendency are similar to those involved in addictive disorders. Under normal circumstances, the reflective system is in charge of the impulsive system in a top-down manner through a variety of mechanisms. However, overactivation in the impulsive system can get rid off the regulation of the reflective system, causing impulsive behavior abnormalities. Bechara (2005) proposed that attractants trigger bottom-up, unconscious signals that regulate or even redirect the cognitive resources from the routine operation and willpower. In addition to the control of the impulsive system by the reflective system, the way people think through the reflective system is also important. Critical thinking can be described as a kind of rational, reflective thinking that centered at making decisions according to behaviors and beliefs (Ennis, 2008). The development of critical thinking skills is essential for students' cognitive and intellectual development (Ennis, 2008; McBride, Xiang, \& Wittenburg, 2002; Stapleton, 2011). High critical thinking is associated with logical analysis to make wise decisions and confidence in their judges (Facione \& Facione, 1990; Lai, 2011). Some studies have found that critical thinking is negatively related to students' social media addiction (Thomas, 2020). Other studies have found that effective training in critical thinking can improve students' attitudes towards substance abuse (Khalili, 2011). Considering the close associations between cognitive features in critical thinking and IA tendency, we presumed that higher critical thinking would link to lower IA tendency.

Taken together, we endeavor to elucidate the GMD differences in IA combining with VBM and to explore the relation between critical thinking and the IA tendency based on the hypothesis that critical thinking will plays a moderating role on the brain structure and IA tendency.

\section{METHODS}

\section{Participants}

Two hundred sixty undergraduates and graduate students participated in the study as part of co-author' project which aimed to establish a long-term longitudinal brain data repository and to explore the correlation between behavior and brain. Because sixteen participants were only collected critical thinking data in the questionnaire acquisition process, 244 participants were considered for formal analysis, including 113 men and 131 women (mean age $=20.02$ years, standard deviation $=1.19$ ). They were asked to fill in the questionnaires measuring the critical thinking and IA 
tendency and then complete MRI scanning. Participants were all right-handed, and reported with no mental illness histories and head motion during scanning were excluded.

\section{Measures}

Measurement of internet addiction tendency. IA tendency was measured by a questionnaire named the Internet Addiction Test (IAT), which was compiled by Young (1998). By summarizing 496 cases of internet overuse, Young revised the IAD identification standard according to the DSM-IV (Young, 1998). Later, Young included an revised version of the IAT, which was aimed to measure the IA tendency in normal people (Young, 1998).This measure includes a total of 20 self-report items, which are divided into four dimensions: "problems", "attachment", "cognitive bias" and "out of control". The participants need to give the items scores on a five-point Likert scale $(1=$ almost never, 5 = always). After the completion of the questionnaire, we calculated the average total scores across participants. The Cronbach coefficient for this measure was 0.90 .

Measurement of critical thinking. California Critical Thinking Disposition Inventory (CCTDI) was provided to measure participants' critical thinking disposition. The inventory has been proven to have good reliability and validity, and the revised Chinese version from Nanjing Normal University (Luo \& Yang, 2001) was used in the current study. The CCTDI included a total of 70 items, which were divided into seven dimensions: truth-seeking (e.g., If there are four reasons to agree with something and only one reason to oppose it, I will choose to agree with it), open mindedness (e.g., I'm trying to be less subjective), analyticity (e.g., I feel anxious when others use shallow arguments to convey good ideas), systematicity (e.g., It's easy for me to organize my thoughts), self-confidence (e.g., I appreciate being able to think accurately), inquisitiveness (e.g., When faced with an important decision, I will do my best to collect all relevant information), and maturity (e.g., The so-called truth is nothing more than an individual's opinion). The participants need to give the items scores on a 6-point Likert scale $(1=$ totally disagree, $6=$ totally agree). After the completion of the questionnaire, we calculated the total scores and subscale scores for the critical thinking of each participant. If the participants scored higher, they had stronger critical thinking disposition. The Cronbach coefficient for this inventory was 0.86 .

\section{Procedure}

All MRI data were acquired on a 3.0-T Siemens Trios MRI scanner (Siemens Medical Systems, Erlangen, Germany), and equipped with an 8-channel radio frequency coil. And we obtained T1-weighted high-resolution anatomical images through a magnetization-prepared rapid acquisition gradient echo (MPRAGE) sequence. The scanning parameters were as follows: repetition time $(\mathrm{TR})=1900 \mathrm{~ms}$; echo time $(\mathrm{TE})=2.52 \mathrm{~ms}$; inversion time $(\mathrm{TI})=900 \mathrm{~ms}$; field of view $(F O V)=256 \times 256$; flip angle $(F A)=9^{\circ}$; slice thickness $=1.0 \mathrm{~mm}$; slices $=176$; voxel size $=1^{*} 1^{*} 1 \mathrm{~mm}^{3}$.

MRI preprocessing based on voxel shape measurements was performed based on SPM8 software (Welcome Trust Center for Neuroimaging, London, UK, http://www.fil.ion. ucl.ac.uk/spm/software/spm8) plugged in MATLAB 7.8 (MathWorks, Natick, Massachusetts, USA). In the first step, images from each participant were manually reoriented and set to the anterior-posterior commissure (AC-PC) for the subsequent analysis images registration. In the second step, we used the new segmentation to separate the images into different parts including gray matter (GM), white matter (WM) and cerebrospinal fluid (CSF). In the third step, we calculated the affine transformation matrix for each image, and then generated roughly aligned image. Next, preprocessing including registration, normalization, and modulation was performed by Diffeomorphic Anatomical Registration through Exponentiated Lie (DARTEL) toolbox based on SPM8, which was used for registration, normalization, and modulation. For GM density (GMD) and WM density (WMD), the warped GM and WM images were obtained through calculating the density change in the voxels (MacDonald, Avis, \& Evans, 1994; Sowell et al., 2002; Thompson et al., 2001). Finally, the images were normalized to Montreal Neurological Institute space (MNI), and then were smoothed by an $8-\mathrm{mm}$ full width and half maximum Gaussian kernel.

\section{Statistical analysis}

The whole-brain MRI analysis was performed in SPM8 software package. And multiple linear models were used to investigate the associations between GMD and IA tendency. In the model, age, gender and whole brain gray matter volume were considered as covariates to control the influence from irrelevant factors. And an masking with a absolute threshold at 0.2 was used to control the edge error generated by the blurred borderline between the GM and WM.

For the multiple regression analysis, we used non-stationarity cluster extent correction (NS toolbox plugged in SPM) to ensure the validity of the result. Non-stationarity is a problem that VBM data needed attentions. This correction was based on the random field theory (RFT), and it considered the size inference under non-stationarity. Ns correction is certified to use in VBM data (Hayasaka, Phan, Liberzon, Worsley, \& Nichols, 2004). And for ns correction, a lower threshold is associated with a more conservative standard (Hayasaka et al., 2004). In the present study, ns correction level was set at $P<0.05$ (Hayasaka et al., 2004), and non-corrected level was set at $P<0.001$.

Behavioral analysis was performed by using SPSS 25.0 (http://www.processmacro.org/index.html), and the further moderation analysis was used the PROCESS in SPSS 25.0. All data were normalized in the correlation and regression analysis. The GMD in the left TPJ was considered as the independent variable, IAT scores were entered as the outcome, and CCTDI scores were the moderator. 


\section{Ethics}

All participants signed the informed consent form and were told that they had the right to withdraw from the study if they did not want to continue. The experiment and scanning process was agreed by the Institutional Review Board (IRB) of co-author's institution (IRB number: H19041).

\section{RESULTS}

\section{Behavioral results}

We examined the Pearson correlation matrix between IAT scores and CCTDI total/subscale scores and found a

Table 1. Descriptive statistics of participant demographics and behavioral measures $(N=244)$

\begin{tabular}{lrrr}
\hline Measure & Mean & SD & Range \\
\hline Age & 20.02 & 1.19 & $18-27$ \\
IAT & 40.44 & 12.62 & $20-79$ \\
CCTDI & 283.23 & 28.22 & $219-376$ \\
Truth-Seeking & 37.38 & 6.76 & $16-55$ \\
Open Mindedness & 40.99 & 5.03 & $27-57$ \\
Analyticity & 42.97 & 4.96 & $28-60$ \\
Systematicity & 38.28 & 5.95 & $22-56$ \\
Self-confidence & 40.34 & 6.31 & $24-60$ \\
Inquisitiveness & 42.95 & 6.34 & $23-60$ \\
Maturity & 40.49 & 6.24 & $22-54$ \\
\hline
\end{tabular}

significant negative correlation between IAT scores and CCTDI scores $(r=-0.356, P<0.001)$. CCTDI subscale scores also exhibited negative correlations with IAT scores. Table 1 shows the descriptive statistics of behavioral data.

\section{Neuroimaging results}

A multiple linear regression model was performed to study the association between GMD and IAT scores while controlling gender, age and whole-brain gray matter volume. The results showed that IAT scores had a significant correlation with GMD in the left temporal-parietal junction (TPJ) (BA:39 x, y, z $=-57,-56,32, t(242)=4.37, P$ (corr) $<0.05)$. As Table 2 and Fig. 1 show, greater GMD in the left TPJ was associated with greater IA tendency (Fig. 1).

\section{Moderation model}

The moderating effect of critical thinking on the relationship between GMD and IA tendency was subsequently tested. First, we calculated a Pearson correlation coefficient between GMD values in the left TPJ, IAT scores and CCTDI scores. The results presented that there were a positive correlation between GMD in the left TPJ and IAT scores $(r=0.307$, $P<0.001)$, negative relation between IAT scores and CCTDI scores $(r=-0.356, P<0.001)$, and CCTDI scores and GMD in the TPJ $(r=-0.137, P<0.05)$.

Then, we used the PROCESS plug-in in SPSS 25.0 to test the moderating effect of critical thinking. The GMD in the

Table 2. Brain regions whose gray matter density is significantly correlated with internet addiction tendency

\begin{tabular}{|c|c|c|c|c|c|c|}
\hline \multirow[b]{2}{*}{ Region } & \multirow[b]{2}{*}{ BA } & \multicolumn{3}{|c|}{ MNI coordinates } & \multicolumn{2}{|c|}{ Cluster size } \\
\hline & & $\mathrm{X}$ & $\mathrm{Y}$ & $\mathrm{Z}$ & $\mathrm{k}$ (voxels) & T-score \\
\hline Positive correlation left TPJ & 39 & -57 & -56 & 32 & 260 & 4.37 \\
\hline
\end{tabular}

Note: $\mathrm{BA}=$ Brodmann area. MNI $=$ Montreal Neurological Institute, voxel size $=1 \mathrm{~mm} \times 1 \mathrm{~mm} \times 1 \mathrm{~mm}, k=$ cluster size. All T-scores reflect a threshold of $P<0.001(P<0.05$, non-stationarity corrected).

$\mathbf{A}$

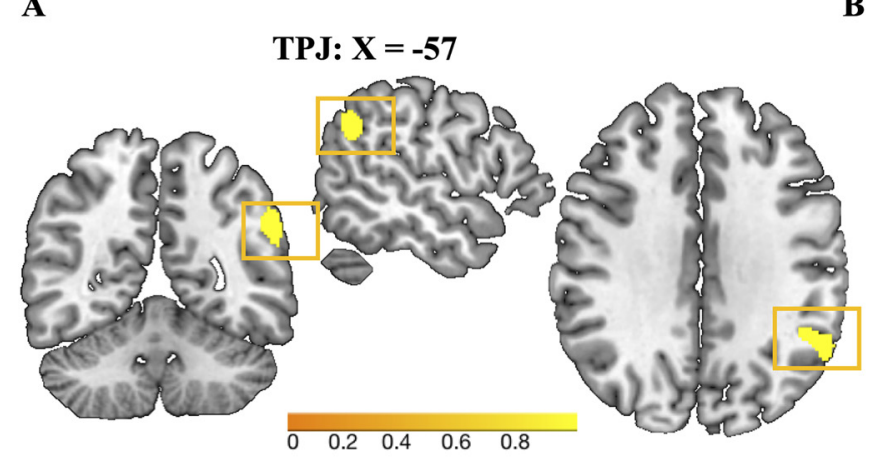

B

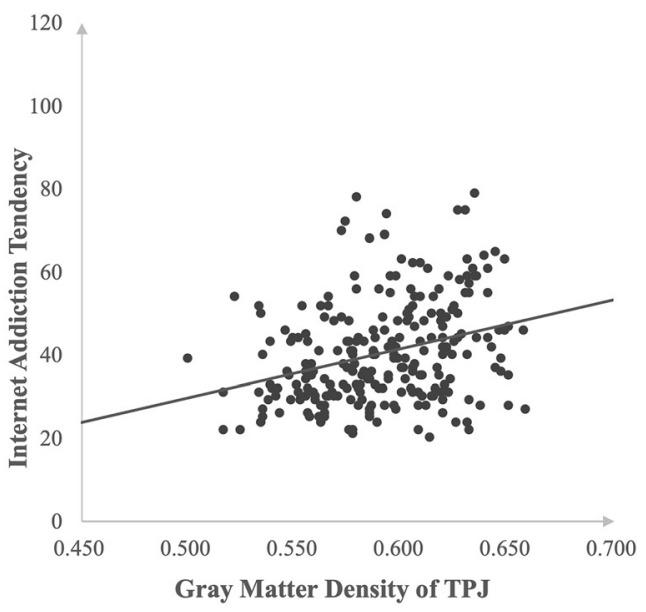

Fig. 1. A. For individuals with higher critical thinking, there is a strong positive correlation between gray matter density (GMD) in the temporal-parietal junction (TPJ) and Internet Addiction Tendency (BA: 39, $\mathrm{x}, \mathrm{y}, \mathrm{z}=-57,-56,32, t(242)=4.37, P$ (corr) $<0.05$, nonstationary corrected). B. Scatterplot of the correlation between GMD and Internet Addiction Tendency. The x-axis represents the regional gray matter density in the TPJ. The y-axis represents the IAT score. The correlation results are shown as $r=0.307, P<0.001$ 
A

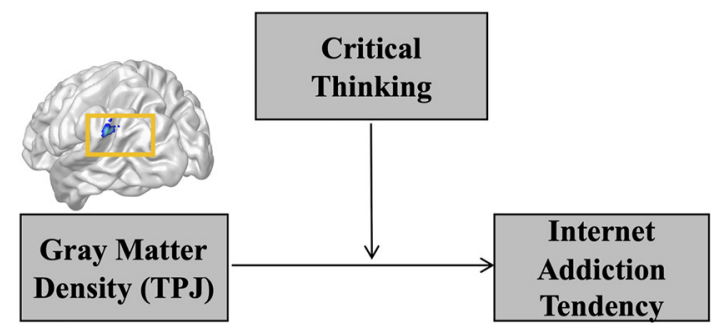

B

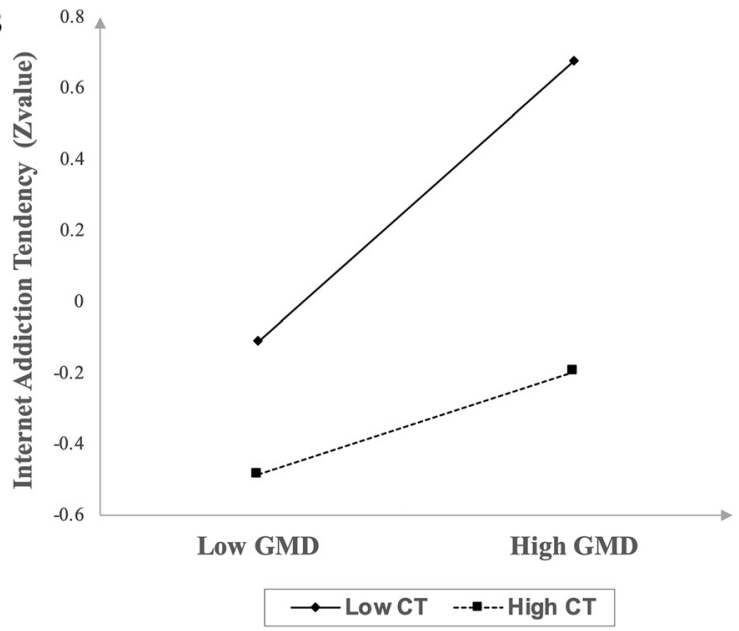

Fig. 2. A. The level of critical thinking (low CT, high CT) moderates the relationship between the GMD in the left temporal-parietal junction (TPJ) and internet addiction tendency. B. For individuals with lower critical thinking, there is a positive correlation between GMD of the TPJ and IAT scores

TPJ values were used as the independent variable, IAT scores as the outcome, and CCTDI scores as the moderator. The results showed a significant moderating effect in the critical thinking between GMD in the TPJ and IA tendency, $P<0.05$, bootstrapped $95 \% C I=-3.3286,-0.1111$, $\mathrm{R}^{2}=0.20$. Figure $2 \mathrm{~A}$ show that critical thinking moderates the link between GMD in the TPJ and IAT scores. For individuals with lower critical thinking abilities, there is a positive correlation between GMD in the TPJ and IAT scores, but for individuals with higher critical thinking, the correlation becomes weaker (Fig. 2B).

\section{DISCUSSION}

This study explores the mechanism of IA tendency and the role of critical thinking disposition from a basic perspective. In accord with the hypothesis, GMD in the TPJ was observed a positive relation with IAT scores. Specifically, the participants with higher IA tendency showed a relatively greater GMD in the TPJ than those with lower IA tendency. In addition, a moderating effect was revealed with critical thinking between GMD in the TPJ and IA tendency. In the individuals with lower critical thinking, those with higher GMD in the TPJ appear more likely to have an IA.

On these grounds, we arrived at the conclusions that IA is characterized by an imbalance between impulsive systems and reflective systems and attention impairments (Bechara, 2005). The TPJ is anatomically located in the junction of the temporal and parietal lobes, which structurally and functionally connects the relevant cortical areas in the temporal lobe and parietal lobe. As an important node in the VAN, the TPJ participates in the integration of complex information from the thalamus and the limbic system and the attention orientation control driven by the somatosensory system and visual and auditory stimuli. Meanwhile, the TPJ is associated with collecting and processing all the information involved in the attention process (Abu-Akel \& Shamay-Tsoory, 2011). Previous studies have demonstrated that TPJ dysfunction occurred in some functional disorders. Sehm et al. (2011) explored the structural features of GM based on VBM in amnesia patients and found that a significant reduction in the GM volume in the right TPJ may result in the inability of amnesia patients to complete working memory tasks (Sehm et al., 2011). Salmon et al. (2005) found that cognitive dysfunction with Alzheimer was related to the weakened strength of the functional connection between the TPJ and superior frontal gyrus. Based on these findings, we may conclude that the TPJ is critical for the maintenance of attention in working memory and thinking processes. The mechanism by which the TPJ participates in addict to the internet and leads to attention dysfunction may be because the TPJ is not only an important part of the attention network but also a central node in the ventral attentional network and participates in "bottomup" cognition, which has a close connection with the control and monitoring of the impulsive system and reflective system mentioned above (Bechara, 2005).

From the neurocognitive perspective, when people engage with the internet, they need willpower coming from the constant exchange of two system through brain structures: an impulsive system responsible for processing the impulsive related emotional information and a reflective system in charge of processing the cognitive control of longterm outcomes. Before one reaches the state that internet indulgence make brain pattern changes, there is a thinking process that the participant needs to undergo. People are required to balance the impulsive and reflective systems to restrain the temptation of the internet and take action. Other studies have demonstrated that clues to addictions stimulate bottom-up processing in addicts, which subsequently change top-down controlling system including attention control (Noël et al., 2005). The TPJ may be an important balance node that captures affective information from the amygdala 
(impulsive system) and simultaneously receives decisions made by the ventral medial prefrontal cortex (reflective system). Thus, the increased GMD in the TPJ may be indicative of greater imbalance between two systems, which means that they are easily to get internet addicted.

According to the results, we can infer that there is a significant link between TPJ and IA performance, and the mechanism of the TPJ is very essential for explaining IA tendency. However, the existence of this relationship is conditional, as we observed that the relationship between the GMD in the TPJ and IA tendency was moderated by critical thinking disposition. Based on previous studies, critical thinking was indeed closely related to IA, and the training of critical thinking was shown to help reduce the IA-related behavior in youth (Thomas, 2020). From Bechara's viewpoint, the formation of addiction is due to excessive activation of the impulsive system, which makes the reflective system ineffective. Critical thinking as an effective thinking skill plays an important role in reflective systems. Mahmoud (2012) indicated a relation between critical thinking and reflective learning styles in students, which means that individuals who have a critical attitude are good at retrieving and integrating information. Moreover, Atay and Karabacak (2012) guided students to use mind mapping and showed that this method improved critical thinking disposition, suggesting superior effects in memory and information integration, organization, and retrieval. Elder and Paul (2013) confirmed that high critical thinking were related to using their rational knowledge to analyze whole thing and compare their thoughts with others. Therefore, critical thinking plays a more promoting role in the reflective system to integrate and convey information in memory in a reasonable and objective form. For low critical thinker, this relationship will be broken, and the impulsive system will directly dominate the process of addiction. The present results showed that individuals with higher critical thinking dispositions were more rational in dealing with information through reflective systems, which may indicate greater resistance to the impulsive system and thus not being addicted to the internet. Therefore, critical thinking disposition exhibited a moderation effect on IA tendency, and good critical thinkers were better at resisting IA. This study provides a more basic perspective on IA and reveals that the variations in susceptibility to IA may be based on critical thinking.

Future studies can be improved in some aspects, such as strengthening the representativeness of samples, choosing actual subjects with prominent symptoms of IA, and comparing the different brain mechanisms of different subtypes of people, such as game addiction, online shopping addiction, and computer addiction. In addition, since there is a strong co-morbidity between ADHD and IAD. ADHD symptoms or relationships with IA need to be considered and emphasized before testing and in future studies to deepen our understanding of IA. Finally, the relationship between the mechanisms of the TPJ and IA tendency is conditional, which suggests that there may be other factors that explain IA performance.

\section{CONCLUSION}

The current study reveals the neural basis of individual differences on internet addiction tendency is the gray matter density differences of temporal-parietal junction. And the relation is moderated by individual critical thinking disposition. Lower critical thinking plays a significant role in the relation between GMD and IA tendency. Nevertheless, individuals with high critical thinking are not easily get addicted and influenced by brain structural discrepancy.

Funding sources: This research was supported by the National Natural Science Foundation of China (32071080; 31571138; 31470981; 31571137; 31500885; 31600878; 31771231), Project of the National Defense Science and Technology Innovation Special Zone, Chang Jiang Scholars Program, National Outstanding Young People Plan, the Program for the Top Young Talents by Chongqing, the Fundamental Research Funds for the Central Universities (SWU1609177), Natural Science Foundation of Chongqing (cstc2015jcyjA10106), Fok Ying Tung Education Foundation (151023), the Research Program Funds of the Collaborative Innovation Center of Assessment toward Basic Education Quality at Beijing Normal University.

Authors' contribution: Di Liu: Conceptualization, Formal analysis, Methodology, Visualization, Writing-original draft; review\&editing. Yuhe Ma: Conceptualization, Formal analysis, Methodology,Writing-original draft. Kaixiang Zhuang: Investigation, Data curation, Methodology, Software. Qunlin Chen: Methodology, Software. Baoguo Shi: Writing-review \& editing, Funding Acquisition, Supervision, Project Administration. Jiang Qiu: Funding Acquisition, Resources, Project Administration, Supervision.

Conflict of interests: The authors declare that they have no conflicts of interest.

\section{REFERENCES}

Abu-Akel, A., \& Shamay-Tsoory, S. (2011). Neuroanatomical and neurochemical bases of theory of mind. Neuropsychologia, 49(11), 2971-2984. https://doi.org/10.1016/j.neuropsychologia. 2011.07.012.

Atay, S., \& Karabacak, Ü. (2012). Care plans using concept maps and their effects on the critical thinking dispositions of nursing students. International Journal of Nursing Practice, 18(3), 233239. https://doi.org/10.1111/j.1440-172x.2012.02034.x.

Bechara, A. (2005). Decision making, impulse control and loss of willpower to resist drugs: A neurocognitive perspective. Nature Neuroscience, 8(11), 1458-1463. https://doi.org/10.1038/nn1584.

Corbetta, M., \& Shulman, G. L. (2002). Control of goal-directed and stimulus-driven attention in the brain. Nature Reviews Neuroscience, 3(3), 201-215. https://doi.org/10.1038/nrn755. 
Dong, G., Lu, Q., Zhou, H., \& Zhao, X. (2011). Precursor or Sequela: Pathological disorders in people with internet addiction disorder. Plos One, 6(2), e14703. https://doi.org/10.1371/ journal.pone.0014703.

Elder, L., \& Paul, R. (2013). Critical thinking: Intellectual standards essential to reasoning well within every domain of thought. Journal of Developmental Education, 36(3), 34-35.

Ennis, R. H. (2008). Nationwide testing of critical thinking for higher education. Teaching Philosophy, 31(1), 1-26. https://doi. org/10.5840/teachphil20083111.

Facione, P. A., \& Facione, N. C. (1990). Critical thinking ability: A measurement tool. Assessment Update, 6(6), 12-13. https://doi. org/10.1002/au.3650060611.

Gennatas, E. D., Avants, B. B., Wolf, D. H., Satterthwaite, T. D., Ruparel, K., Ciric, R., ... Gur, R. C. (2017). Age-related effects and sex differences in gray matter density, volume, mass, and cortical thickness from childhood to young adulthood. The Journal of Neuroscience, 37(20), 5065-5073. https://doi.org/10. 1523/jneurosci.3550-16.2017.

Gur, R. C., Butler, E. R., Moore, T. M., Rosen, A. F., Ruparel, K., Satterthwaite, T. D., \& Gur, R. E. (2021). Structural and functional brain parameters related to cognitive performance across development: Replication and extension of the parieto-frontal integration theory in a single sample. Cerebral Cortex, 31(3), 1444-1463. https://doi.org/10.1093/cercor/bhaa282.

Hayasaka, S., Phan, K. L., Liberzon, I., Worsley, K. J., \& Nichols, T. E. (2004). Nonstationary cluster-size inference with random field and permutation methods. Neuroimage, 22(2), 676-687. https://doi.org/10.1016/j.neuroimage.2004. 01.041.

Holden, C. (2001). 'Behavioral' addictions: Do they exist? Science, 294(5544), 980-982. https://doi.org/10.1126/science.294.5544. 980.

Khalili, A., Sohrabi, F., \& Afkhami, M. (2011). The effectiveness of training critical thinking skills on students' attitude towards substance abuse. Research on Addiction, 5(17), 91-106.

Kühn, S., \& Gallinat, J. (2014). Brains online: Structural and functional correlates of habitual internet use. Addiction Biology, 20(2), 415-422. https://doi.org/10.1111/adb.12128.

Kuss, D. J., \& Lopez-Fernandez, O. (2016). Internet addiction and problematic internet use: A systematic review of clinical research. World Journal of Psychiatry, 6(1), 143-176. https:// doi.org/10.5498/wjp.v6.i1.143.

Lai, E. R. (2011). Critical thinking: A literature review. New York, NY: Pearson's Publications.

Li, W., Li, Y., Yang, W., Zhang, Q., Wei, D., Li, W., ... Qiu, J. (2015). Brain structures and functional connectivity associated with individual differences in Internet tendency in healthy young adults. Neuropsychologia, 70, 134-144. https://doi.org/10. 1016/j.neuropsychologia.2015.02.019.

Luo, Q. X., \& Yang, X. H. (2001). Revision for CCIDI (Chinese version). Psychological Development and Education, 17(3), 47-51. https://doi.org/10.3969/j.issn.1001-4918.2001.03.009.

MacDonald, D., Avis, D., \& Evans, A. (1994). Multiple surface identification and matching in magnetic resonance images. In R. A. Robb (Ed.), Proceedings of the SPIE conference on visualization in biomedical computing (pp. 160-169). Rochester, MN: SPIE.
Mahmoud, H. G. (2012). Critical thinking dispositions and learning styles of Baccalaureate nursing students and its relation to their achievement. International Journal of Learning and Development, 2(1), 398-415. https://doi.org/10.5296/ijld.v2i1.1379.

McBride, R. E., Xiang, P., \& Wittenburg, D. (2002). Dispositions toward critical thinking: The preservice teacher's perspective. Teachers and Teaching, 8(1), 29-40. https://doi.org/10.1080/ 13540600120110556.

Müller, K. W., Beutel, M. E., \& Wölfling, K. (2013). 984 - Psychopathological symptoms and co-morbid disorders associated with internet addiction and pathological gambling: Analyses of a clinical sample. European Psychiatry, 28, 1.https://doi.org/10. 1016/s0924-9338(13)76120-0.

Noël, X., Van der Linden, M., d'Acremont, M., Colmant, M., Hanak, C., Pelc, I., .. Bechara, A. (2005). Cognitive biases toward alcohol-related words and executive deficits in polysubstance abusers with alcoholism. Addiction, 100(9), 13021309. https://doi.org/10.1111/j.1360-0443.2005.01125.x.

Salmon, E., Ruby, P., Perani, D., Kalbe, E., Laureys, S., Adam, S., \& Collette, F. (2005). Two aspects of impaired consciousness in Alzheimer's disease. Progress in Brain Research, 287-298. https://doi.org/10.1016/s0079-6123(05)50021-9.

Sehm, B., Frisch, S., Thöne-Otto, A., Horstmann, A., Villringer, A., \& Obrig, H. (2011). Focal retrograde amnesia: Voxelbased morphometry findings in a case without MRI lesions. Plos One, 6(10), e26538. https://doi.org/10.1371/journal.pone. 0026538.

Sowell, E. R., Thompson, P. M., Rex, D., Kornsand, D., Tessner, K. D., Jernigan, T. L., \& Toga, A. W. (2002). Mapping sulcal pattern asymmetry and local cortical surface gray matter distribution in vivo: Maturation in perisylvian cortices. Cerebral Cortex, 12(1), 17-26. https://doi.org/10.1093/cercor/12. 1.17 .

Stapleton, P. (2011). A survey of attitudes towards critical thinking among Hong Kong secondary school teachers: Implications for policy change. Thinking Skills and Creativity, 6(1), 14-23. https://doi.org/10.1016/j.tsc.2010.11.002.

Thomas, D. (2020). Social media addiction, critical thinking and achievement emotions among EFL students in Thailand. Asia Pacific Journal of Educators and Education, 35(1), 157-171. https://doi.org/10.21315/apjee2020.35.1.9.

Thompson, P. M., Mega, M. S., Woods, R. P., Zoumalan, C. I., Lindshield, C. J., Blanton, R. E., .. . Toga, A. W. (2001). Cortical change in Alzheimer's disease detected with a disease-specific population-based brain atlas. Cerebral Cortex, 11(1), 1-16. https://doi.org/10.1093/cercor/11.1.1.

Wang, C. W., Ho, R. T., Chan, C. L., \& Tse, S. (2015). Exploring personality characteristics of Chinese adolescents with internetrelated addictive behaviors: Trait differences for gaming addiction and social networking addiction. Addictive Behaviors, 42, 32-35. https://doi.org/10.1016/j.addbeh.2014.10.039.

Weinstein, A., \& Lejoyeux, M. (2010). Internet addiction or excessive internet use. The American Journal of Drug and Alcohol Abuse, 36(5), 277-283. https://doi.org/10.3109/ 00952990.2010.491880.

Xu, J., Shen, L. X., Yan, C. H., Hu, H., Yang, F., Wang, L., ... Shen, X. M. (2012). Personal characteristics related to the risk of adolescent internet addiction: A survey in Shanghai, China. 
BMC Public Health, 12(1), 1106. https://doi.org/10.1186/1471 2458-12-1106.

Yen, J. Y., Ko, C. H., Yen, C. F., Wu, H. Y., \& Yang, M. J. (2007). The comorbid psychiatric symptoms of internet addiction: Attention deficit and hyperactivity disorder (ADHD), depression, social phobia, and hostility. Journal of Adolescent Health, 41(1), 93-98. https://doi.org/10.1016/j.jadohealth. 2007.02.002.
Young, K. S. (1998). Internet addiction: The emergence of a new clinical disorder. CyberPsychology \& Behavior, 1(3), 237-244. https://doi.org/10.1089/cpb.1998.1.237.

Yücens, B., \& Üzer, A. (2018). The relationship between internet addiction, social anxiety, impulsivity, self-esteem, and depression in a sample of Turkish undergraduate medical students. Psychiatry Research, 267, 313-318. https://doi.org/10.1016/j. psychres.2018.06.033. 